\title{
ORIGINAL
}

\section{VALIDACIÓN DE LA CAUSA BÁSICA DE DEFUNCIÓN EN LAS MUERTES QUE REQUIEREN INTERVENCIÓN MEDICOLEGAL}

\author{
Mercè Gotsens (1,2,3), Marc Marí-Dell'Olmo (1,2,3), Maica Rodríguez-Sanz $(1,2,3)$, \\ Dolores Martos (2,3), Albert Espelt $(1,2,3)$, Glòria Pérez $(1,2,3,4)$, Katherine Pérez $(1,2,3)$, \\ M Teresa Brugal $(1,2,3)$, Eneko Barbería Marcalain (5) y Carme Borrell $(1,2,3,4)$
}

(1) CIBER Epidemiología y Salud Pública (CIBERESP)

(2) Agència de Salut Pública de Barcelona.

(3) Institut d'Investigació Biomèdica (IIB Sant Pau). Barcelona.

(4) Universitat Pompeu Fabra. Barcelona.

(5) Institut de Medicina Legal de Catalunya. Departament de Justícia. Barcelona.

\section{RESUMEN}

Fundamento: Las defunciones por causas externas requieren exámenes complementarios para determinar la causa de muerte. Si no se incorporan estos resultados a los registros de mortalidad estas causas pueden quedar mal clasificadas. El objetivo del estudio es validar la causa básica de defunción del Registro de Mortalidad con la obtenida de las fuentes forenses, en Barcelona entre los años 2004 y 2006.

Métodos: Diseño transversal. La población de estudio son los fallecidos residentes en Barcelona con intervención medicolegal entre 2004 y 2006. Las fuentes de información son el Registro de Mortalidad y el archivo de patología forense del Instituto de Medicina Legal de Catalunya (IMLC) (estándar de comparación). Las variables son la causa de defunción, el sexo y la edad. Se calcula la sensibilidad, el porcentaje de confirmación (PC) y sus intervalos de confianza al 95\% (IC95\%).

Resultados: La sensibilidad de las causas externas es 59,7\% (IC95\%:56,5-62,9) y el PC 96,7\% (IC95\%:94,8-98,0). Las lesiones por tráfico, las intoxicaciones y los suicidios están subnotificados en el Registro de Mortalidad siendo la sensibilidad inferior al $45 \%$ y el PC superior al $80 \%$. Las causas mal definidas están sobrenotificadas siendo la sensibilidad de $89,2 \%$ (IC95\%:83,4-93,4) y el PC de $28,0 \%$ (IC95\%:24,2$32,1)$. No hay diferencias por sexo y edad.

Conclusiones: La validez de las causas externas del Registro de Mortalidad es escasa por la subnotificación y el elevado porcentaje de causas mal definidas. Según los resultados, incorporar la información de las fuentes forenses al Registro de Mortalidad aumenta la calidad de las estadísticas de mortalidad.

Palabras clave: Mortalidad. Causas externas. Autopsia. Validación.

\section{ABSTRACT}

\section{Validation of the Underlying Cause of Death in Medicolegal Deaths}

Background: Deaths due to external causes require additional medical tests in order to determine the cause of death. If these results are not incorporated into the death register these causes may be misclassified. The objective of this study is to validate the underlying cause of death of the Mortality Register with information obtained from forensic sources in Barcelona between 2004 and 2006.

Methods: Cross-sectional design. The study population consisted of deceased residents in Barcelona with a medicolegal intervention between 2004 and 2006. The sources of information are the Mortality Registry and the forensic pathology file filled in by Institute of Legal Medicine of Catalonia (ILMC) (gold standard). The study variables are the cause of death, sex and age. Sensitivity and percentage of confirmation (PC) with $95 \%$ confidence intervals $(95 \% \mathrm{CI})$ are calculated.

Results: The sensitivity of external causes is $59.7 \%$ (95\% CI:56.5-62.9) and PC is $96.7 \%$ (95\% CI:94.8-98.0). Traffic injuries, poisonings and suicides are under-reported in the Mortality Register with a sensitivity lower than $45 \%$ and a PC higher than $80 \%$. Symptoms, signs and ill-defined conditions are over-reported with a sensitivity of $89.2 \%$ (95\% CI:83.4-93.4) and a PC of $28.0 \%$ (95\% CI:24.2-32.1). There are no differences by sex and age.

Conclusions: The validity of the external causes in the Mortality Register is low due to under-reporting and the high proportion of symptoms signs and ill-defined causes. According to the results, incorporating information from forensic sources to the Mortality Register increases the quality of mortality statistics.

Keywords: Mortality. External causes. Autopsy. Validation.

\footnotetext{
Correspondencia:

Carme Borrell

Agència de Salut Pública de Barcelona

Plaça Lesseps 1

08023 Barcelona

cborrell@aspb.cat
} 


\section{INTRODUCCIÓN}

Las estadísticas de mortalidad son una herramienta ampliamente utilizada en el ámbito de la salud pública. Entre sus usos destaca la ayuda que proporcionan en la planificación sanitaria y en la vigilancia de la salud de la población ${ }^{1}$. En nuestro país estas estadísticas se basan en la información declarada en el Boletín Estadístico de Defunción (BED).

En el caso de las defunciones producidas en circunstancias violentas o sospechosas de criminalidad, el médico no debe emitir el Certificado Médico de Defunción (CMD) y tiene que comunicar el fallecimiento al juzgado de guardia ${ }^{2}$. Estas defunciones son estudiadas por los Institutos de Medicina Legal mediante autopsias medicolegales que tienen como objetivo determinar la causa y circunstancias de la muerte (origen por causas naturales o violentas, identidad de la persona fallecida y fecha de la muerte). Las autopsias medicolegales constan de tres fases: levantamiento del cadáver e investigación de las circunstancias de la muerte, examen del cadáver y exámenes complementarios (toxicológicos e histológicos) ${ }^{3}$.

En España las defunciones que requieren intervención medicolegal son principalmente las relacionadas con los casos obvios o sospechosos de homicidio, suicidio o accidente, clasificadas en el grupo de causas externas, y los casos de muerte súbita, inesperada e inexplicada, clasificadas como causas naturales o mal definidas ${ }^{3,4}$. Hasta 2009 las muertes con intervención medicolegal requerían un documento de declaración adicional, el MNP.52 (Movimiento Natural de Población), cumplimentado a menudo por personal del juzgado, en el que se especificaba el motivo supuesto de la defunción. El MNP.52 y el BED, ambos documentos con finalidad estadística, se remitían al Registro Civil y posteriormente al Instituto Nacional de Estadística (INE) (figura 1). En enero de 2009, con el objetivo de mejorar la calidad de la certificación, el MNP.52 fue sustituido por el Boletín Estadístico de Defunción con Intervención Judicial ${ }^{2}$.

Debido a la inmediatez con que se rellenan los documentos estadísticos es frecuente cumplimentar el MNP.52 antes de disponer de los informes definitivos de autopsia, a pesar de las recomendaciones de la OMS y Eurostat, que establecen que la causa de la muerte debe recogerse del informe definiti$v^{5}{ }^{5}$. En consecuencia, estas causas pueden quedar mal clasificadas en las estadísticas de mortalidad. Existen algunos países, como Finlandia, Noruega y el Reino Unido que incorporan al registro de mortalidad la información procedente de los informes forenses, lo que hace mejorar la validez de las estadísticas de mortalidad ${ }^{6-9}$. Diferentes estudios han validado la mortalidad por causas externas mediante la información médica disponible, encontrando discrepancias entre la causa básica registrada en las estadísticas de mortalidad y la causa obtenida después de los estudios post mórtem ${ }^{10-17}$. En nuestro país destaca el trabajo de De Arán et al. ${ }^{18}$ que comparaba la causa básica de defunción del Registro de Mortalidad de Catalunya con la información medicolegal de las defunciones judiciales, encontrando que la notificación de las causas externas en el Registro de Mortalidad carecía de exactitud.

Desde 1985, en la ciudad de Barcelona se integra en las estadísticas de mortalidad la información referente a las muertes con intervención medicolegal, con el propósito de mejorar la calidad del Registro de Mortalidad de la ciudad. Disponer de la información procedente de los informes forenses y del Registro de Mortalidad permite comparar las dos fuentes de información prestando especial atención a la validez de las causas externas en general, y a las lesiones por tráfico, los suicidios, las intoxicaciones y los homicidios en particular. Por otro lado, debido a que la mortalidad por causas externas es más frecuente entre los hombres y los jóvenes es importante analizar si existen diferencias en la validación 


\section{Figura 1}

\section{Resumen esquemático de la obtención de la información de mortalidad por parte del Registro de Mortalidad de Barcelona hasta 2009}

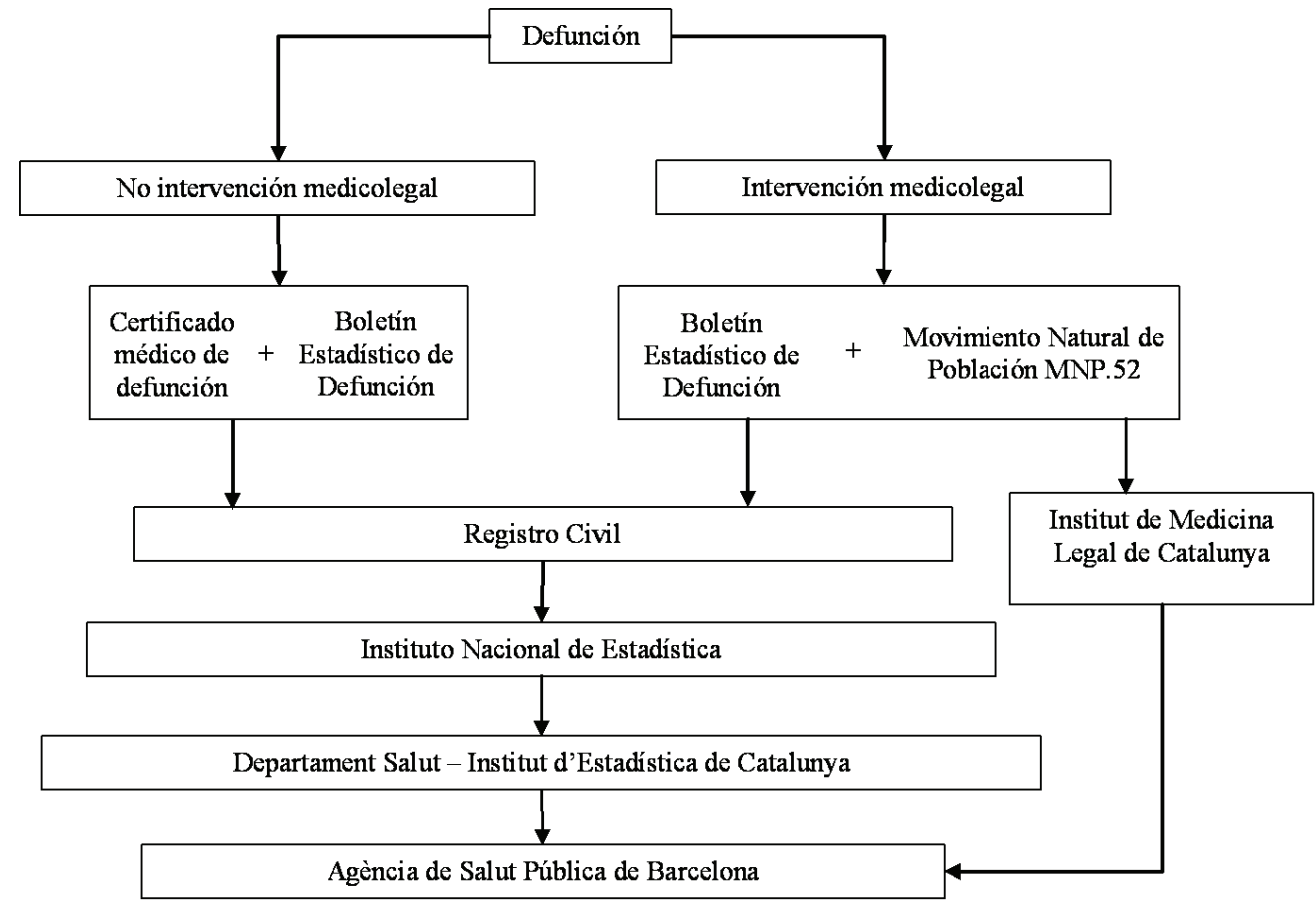

de estas causas según sexo y edad, aspectos que no se han analizado previamente.

El objetivo de este estudio es validar la causa básica de defunción que consta en los documentos estadísticos (BED y MNP.52) con la obtenida a partir de los informes forenses definitivos en las defunciones por causas externas de los residentes en Barcelona que requieren la intervención medicolegal entre los años 2004 y 2006, así como analizar si existen diferencias según sexo y edad.

\section{SUJETOS Y MÉTODO}

Se trata de un estudio de diseño transversal. La población de estudio fueron todas las personas fallecidas en Barcelona, residentes en esta ciudad, con intervención medicolegal entre los años 2004 y 2006. Las fuentes de información utilizadas fueron el Registro de Mortalidad de Catalunya del Departament de Salut de la Generalitat de Catalunya para las causas de defunción recogidas en los documentos estadísticos oficiales y el archivo de patología forense del Institut de Medicina Legal de Catalunya (IMLC) para la información medicolegal, que se consideró como el estándar de comparación. La información medicolegal recogida procedía de fuentes de información diversas, desde la historia clínica aportada por los hospitales (en el caso que la víctima fallezca en un centro hospitalario), testigos presenciales de los hechos, informes de la guardia urbana en los accidentes de tráfico, etcétera, así como de los propios resultados forenses. Un informe forense recoge los antecedentes personales y familiares de la 
persona fallecida, el lugar y las circunstancias de la muerte, el examen externo e interno del cadáver y el resultado de los exámenes complementarios. Las conclusiones del informe forense incluyen el diagnóstico anatomopatológico, el tipo de muerte (natural o violenta), la causa de la defunción y en caso de muerte violenta la etiología medicolegal (homicida, suicida, accidental o indeterminada) ${ }^{19}$. A partir de los informes forenses se obtiene la información necesaria para poder codificar la causa básica de defunción. Para este estudio, se introdujo en una base de datos la causa básica de defunción del IMLC, codificada por una profesional entrenada, y las variables de tomo y página de defunción del Registro Civil como datos identificativos, ya que cada defunción tiene adjudicada una sola página en un tomo.

Se realizó un enlace determinista entre el Registro de Mortalidad y el registro con datos del IMLC utilizando los datos identificativos del tomo y la página del Registro Civil. En total se enlazaron un $97,4 \%$ de defunciones con intervención medicolegal, obteniendo 2.226 muertes con información del IMLC y de la causa básica que consta en el Registro de Mortalidad.

Las variables estudiadas fueron el sexo, la edad agrupada en dos categorías ( $<50$ años y $\geq 50$ años), la causa básica de defunción que consta en el Registro de Mortalidad y la causa básica de defunción que consta en el registro del IMLC. Las causas básicas de defunción fueron codificadas mediante los criterios establecidos por la Clasificación Internacional de Enfermedades décima revisión (CIE10) en 17 grupos basados en los 20 Capítulos de la CIE-10, uniendo en un solo grupo los capítulos correspondientes a las enfermedades del sistema nervioso, enfermedades del ojo y sus anexos y enfermedades del oído y las apófisis mastoides ${ }^{20}$. El grupo de causas externas de mortalidad se desagregó en 5 grupos: lesiones por accidente de tráfico (lesiones por tráfico), intoxicaciones, caídas, suicidios, homicidios y resto de causas externas.
Se realizó un análisis descriptivo para analizar qué porcentaje representan las muertes con intervención medicolegal respecto al total de defunciones de residentes en Barcelona ocurridas en esta ciudad. Posteriormente se realizó un análisis descriptivo de las defunciones obtenidas del Registro de Mortalidad y del IMLC según grupo de causa por sexo y edad. Para el análisis de la concordancia entre la causa básica de defunción del Registro de Mortalidad y la del IMLC se calculó el índice Kappa $^{21}$ con su intervalo de confianza al 95\% y un índice de concordancia simple calculado como el cociente entre el número de defunciones del Registro de Mortalidad que coinciden con las del IMLC y el total de defunciones. El estudio de la validez se centró en las causas externas y mal definidas, con este objetivo se calculó la sensibilidad y el porcentaje de confirmación (PC) con sus respectivos intervalos de confianza al $95 \%$ calculados mediante el método exacto, considerando como estándar de comparación la información procedente del IMLC. Para cada causa, se definió la sensibilidad como el cociente entre el número de defunciones concordantes entre los dos registros y el total de defunciones de esa misma causa registrada en el IMLC. El PC se definió como el cociente entre el número de defunciones concordantes entre los dos registros y el total de defunciones de esa misma causa registrada en el Registro de Mortalidad. Estos indicadores permitieron agrupar las causas en 4 categorías utilizando los criterios de Percy et al. ${ }^{22}$ : i) Causas fiables: cuando la sensibilidad y el PC toman valores iguales o superiores al $80 \%$; ii) Causas con escasa validez: cuando la sensibilidad y el PC toman valores inferiores al $80 \%$; iii) Causas sobrenotificadas: cuando la sensibilidad es mayor que el PC, siendo el $\mathrm{PC}$ inferior al $80 \%$; iv) Causas subnotificadas: cuando la sensibilidad es menor que el PC, siendo la sensibilidad inferior al $80 \%$. Los análisis se estratificaron por sexo y edad. 


\section{RESULTADOS}

Las muertes con intervención medicolegal representaron aproximadamente el 5\% del total de defunciones de residentes en Barcelona producidas en esta ciudad durante el período estudiado. Este porcentaje varió según causa, así el 53,2\% de las causas mal definidas y el $40,8 \%$ de las causas externas codificadas en el Registro de Mortalidad requieren la intervención medicolegal. En el caso de las lesiones por tráfico, las intoxicaciones, los suicidios y los homicidios, los porcentajes son superiores al 90\%. En cambio, sólo el 16,2\% de las caídas requirieron la intervención medicolegal. Para el grupo de causas naturales este porcentaje fue del $2,7 \%$ (tabla 1).

Entre 2004 y 2006 se registraron un total de 2.226 defunciones de residentes en Barcelona que requirieron intervención medicolegal, de las cuales $1.501(67,4 \%)$ fueron hombres y $1.519(68,2 \%)$ fueron personas mayores de 50 años. En la tabla 2 se muestra la distribución de las defunciones con inter- vención medicolegal según sexo y grupos de edad. El 23,7\% de las defunciones quedaron codificadas en el Registro de Mortalidad como causas mal definidas, el $25,6 \%$ dentro del grupo de causas externas y el 50,7\% como causas naturales. Considerando la información del IMLC, el porcentaje de defunciones codificadas como mal definidas se redujo al 7,4\% mientras que el de causas externas aumentó al 41,4\%. En las personas menores de 50 años se observó que según la información del IMLC el porcentaje de defunciones codificadas en el grupo de causas externas fue del $65,5 \%$, siendo las intoxicaciones $(23,2 \%)$ y los suicidios las defunciones más frecuentes $(19,9 \%)$.

Para analizar la concordancia entre la causa básica de defunción que constaba en el Registro de Mortalidad y la obtenida a partir de la información del IMLC se calculó el índice de concordancia global simple para todos los grupos de causas, que fue del $54,8 \%$ (IC95\%: 52,7-56,8) y el valor del índice Kappa que fue del 46,0\% (IC95\%: $44,1-48,0)$.

Tabla 1

Número total de defunciones obtenidas del Registro de Mortalidad (RM) y número y porcentaje de defunciones con intervención medicolegal (IMLC), de residentes en Barcelona ocurridas en esta ciudad según tipo de causa, 2004-2006

\begin{tabular}{|l|l|r|r|r|}
\hline & \multicolumn{1}{|c|}{ CIE-10 } & RM & IMLC & \% IMLC \\
\hline Causas naturales & A00-Q99 & 41.449 & 1.129 & 2,7 \\
\hline Mal definidas & R00-R99 & 992 & 528 & 53,2 \\
\hline Total causas externas & V01-Y98 & 1.396 & 569 & 40,8 \\
\hline Lesiones tráfico & V01-V06(.1), V09(.2-.9), & 52 & 49 & 94,2 \\
& V10-V18(.3-.9), V19(.4-.9), \\
& V20-V28(.3-.9), V29-V79(.4-.9), & & & \\
& V80(.3-.6), V81-V82(.1), & & & \\
& V83-V86(.0-.4), V87(.0-.9), & & & \\
& V89(.2-.9) & & & \\
\hline Intoxicaciones por sustancias & X40-X49 & 51 & 47 & 92,2 \\
\hline Caídas & W00-W19 & 266 & 43 & 16,2 \\
\hline Suicidios & X60-X84 & 156 & 144 & 92,3 \\
\hline Homicidios & X85-X99, Y00-Y09 & 14 & 14 & 100,0 \\
\hline Resto de causas externas & V90-V99, W20-W99, X00-X39, & 857 & 272 & 31,7 \\
\hline XOTAL & X50-X59, Y10-Y36, Y40-Y89 & & & \\
\hline
\end{tabular}




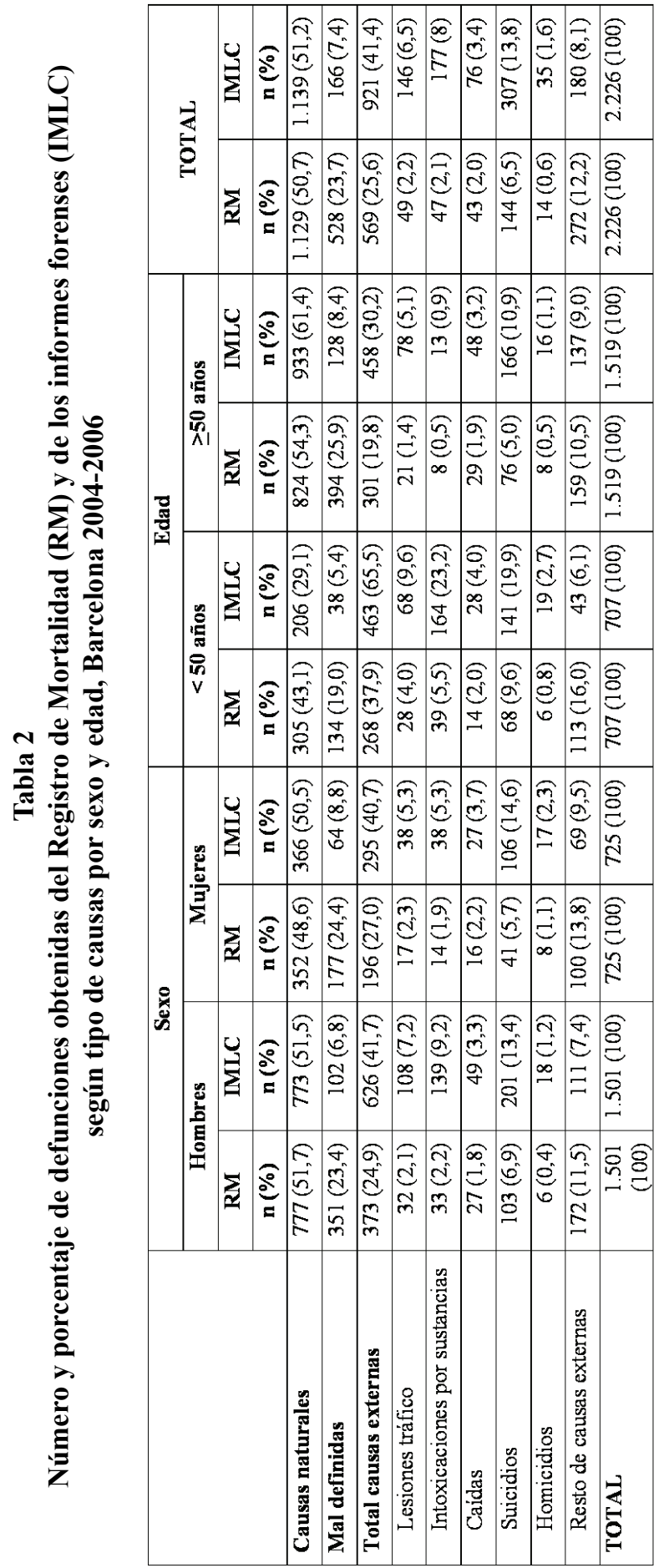




\begin{tabular}{|c|c|c|c|c|c|c|c|c|c|c|c|c|c|c|c|c|c|c|c|c|c|c|c|}
\hline \multirow{3}{*}{ 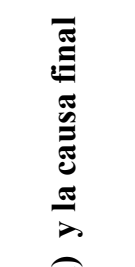 } & 芯 & $\stackrel{N}{N}$ & 이 & $\infty$ & $m$ & 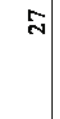 & $\approx$ & 8 & : & : & & -7 & in & & $N$ & & 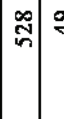 & g & 与 & $m g$ & $\exists$ & $\mathbb{N}$ & 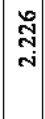 \\
\hline & 点 & in & $m$ & $\rightarrow$ & & - & $N$ & $\div$ & $\stackrel{\infty}{\sim}$ & $m$ & & & & & & & मे & $N$ & $N \mid \alpha$ & \begin{tabular}{l|l}
$\infty$ & $\sim$
\end{tabular} & \begin{tabular}{l|l}
$N$ & $N$
\end{tabular} & $\vec{\infty}$ & $\stackrel{\circ}{\infty}$ \\
\hline & 員 & & & $\rightarrow$ & & & & $N$ & & & & & & & -1 & & 음 & & & - & $=$ & $\Rightarrow a$ & $m$ \\
\hline & '룡 & $\rightarrow$ & $N$ & $m$ & $\rightarrow$ & & $\infty$ & $\pi$ & $\approx$ & $\nabla$ & & & & & & & a & -7 & $\nabla=$ & $=\cong$ & $\approx-$ & $\Phi$ & 용 \\
\hline 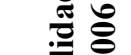 & 명 & $\rightarrow$ & $\mathbf{N}$ & $\rightarrow$ & & & & $m$ & $\nabla$ & & & & & & & & $\infty$ & & & $\exists \cong$ & $\approx$ & 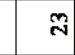 & $\stackrel{\circ}{2}$ \\
\hline 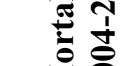 & 司 & & & $\rightarrow$ & & 驺 & $N$ & \pm & m & $m$ & & & & & & & m & & ले & & $m$ & & $E$ \\
\hline 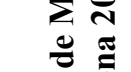 & 夠 & & & & & & & $=$ & $\nabla$ & & & & & & & & $\stackrel{\infty}{\sim}$ & +0 & ? & $\begin{array}{lll}N & -\end{array}$ & -7 & : & $\stackrel{0}{=}$ \\
\hline 远 & 焉 & & -7 & & & & & $n$ & - & -1 & & & $N$ & & & & $\mid \begin{array}{c}\infty \\
=\end{array}$ & & $\rightarrow 7$ & $\rightarrow$ & & & $\stackrel{0}{0}$ \\
\hline$\tilde{0}$ & : & & & & & & & $m$ & - & & & & & & & & & & & & & & $\nabla$ \\
\hline$\stackrel{0}{0}$ & 㤐 & & & & & & & & & & & & & & & & & & & & & & \\
\hline 吾 $\bar{g}$ & 司 & & & & & & & & & & & & & & & & & & & & & & \\
\hline$m$ \& & 氞司 & $m$ & & & & & & $N$ & & & & & N & & & & -7 & & & & & & $\infty$ \\
\hline$\Xi$ & 茄 & & & & & & & & & & & & & & & & & & & & & & \\
\hline : & 蚛 & & & & & & & & & & & & & & & & -7 & & & & & & -7 \\
\hline$\underset{0}{\Xi}$ & 品 & $n$ & & $\rightarrow$ & $\rightarrow$ & & & $a$ & $a$ & 8े & & & & & & & ส & & & - & & $\rightarrow$ & 工 \\
\hline$\underset{\sigma}{\theta} \stackrel{\Xi}{\sigma}$ & 岕 & $N$ & & & & & & 웅 & $\infty$ & & & & & & & & $\infty$ & & -7 & & & $\rightarrow$ & $\mathbb{\Xi}$ \\
\hline  & 号 & $\rightarrow$ & in & & & & & $\begin{array}{c}2 \\
2 \\
\text { n. }\end{array}$ & $m$ & in & & $\rightarrow$ & $\rightarrow$ & & -1 & & 员 & & & $\rightarrow 7$ & -1 & 0 & : \\
\hline$\stackrel{\mathscr{V}}{\Xi}$ & 荧 & & & & & & $m$ & & - & & & & & & & & & & & & & - & $n$ \\
\hline 苋 & 芴 & & & & & & & & - & & & & & & & & $\mathbf{N}$ & & & & & & $m$ \\
\hline 氖 & 固 & & & & -1 & & & $m$ & & -1 & & & & & & & $m$ & & & & & & $\infty$ \\
\hline$\frac{\pi}{0}$ & 唺 & & & & & & & & & $\neg$ & & & & & & & & & & & & & -1 \\
\hline 己ֶ & 具 & & $r$ & & & & & $\infty$ & $n$ & $N$ & & & & & & & $\stackrel{\infty}{\sim}$ & & & & 7 & $N$ & m \\
\hline e & 岕 & 6 & & & & - & & -7 & -7 & -1 & & & & & & & $N$ & & & & 7 & & $m$ \\
\hline 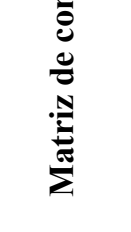 & 幽 &  & 总 & 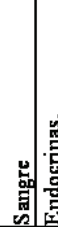 & 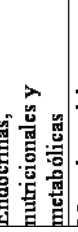 & 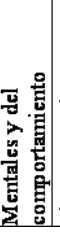 & 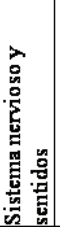 & 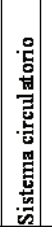 & 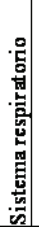 & 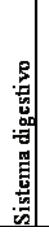 & 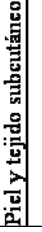 & 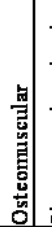 & 占. & 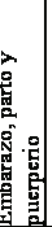 & 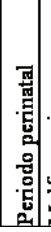 & 总 & 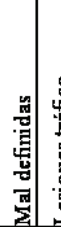 & 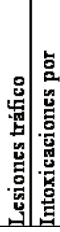 & 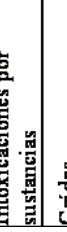 & 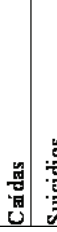 & 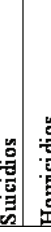 & 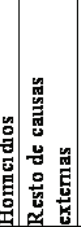 & 包 \\
\hline
\end{tabular}




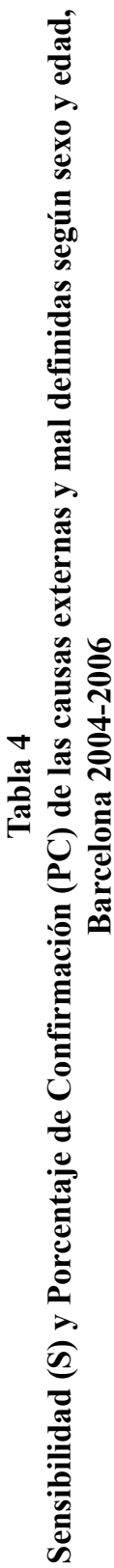

\begin{tabular}{|c|c|c|c|c|c|c|c|c|c|c|c|c|c|}
\hline \multirow{2}{*}{ 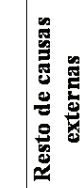 } & $\begin{array}{l}\stackrel{2}{2} \\
2 \\
0 \\
0\end{array}$ & & $\begin{array}{l}a \\
m \\
n \\
i \\
\dot{m} \\
\dot{m}\end{array}$ & $\begin{array}{l}a \\
a \\
\tilde{n} \\
\alpha \\
\hat{\sim} \\
\vec{\sim}\end{array}$ & \begin{tabular}{|l|}
$\infty$ \\
$\infty$ \\
$\infty$ \\
$n$ \\
$m$ \\
$m$ \\
$=$ \\
$m$ \\
$m$
\end{tabular} & 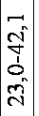 & & 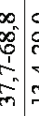 & $\begin{array}{l}0 \\
0 \\
8 \\
y \\
y \\
m^{n}\end{array}$ & $\begin{array}{l}- \\
\vec{n} \\
\dot{\alpha} \\
m \\
m\end{array}$ & 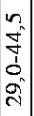 & $\begin{array}{l}0 \\
i \\
n \\
0 \\
0 \\
\tilde{m} \\
n\end{array}$ & $\begin{array}{l}0 \\
w \\
m \\
m \\
\dot{y} \\
\dot{N} \\
\dot{N}\end{array}$ \\
\hline & ¿ & & $\vec{F}$ & $\mid \begin{array}{l}n \\
\infty \\
\infty \\
c\end{array}$ & $\begin{array}{l}\overrightarrow{6} \\
\mathscr{b}^{n}\end{array}$ & $\begin{array}{c}0 \\
N \\
m \\
m\end{array}$ & & $\begin{array}{c}n \\
m \\
n\end{array}$ & $\begin{array}{l}\dot{s}_{n}^{n} \\
\stackrel{N}{ }\end{array}$ & $\begin{array}{c}m \\
\mathrm{y}\end{array}$ & $\begin{array}{l}n \\
0 \\
0 \\
n\end{array}$ & $\begin{array}{l}0 \\
n^{n}\end{array}$ & $\begin{array}{l}\infty \\
\text { on } \\
\text { in }\end{array}$ \\
\hline \multirow{2}{*}{ 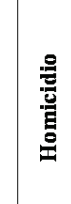 } & $\begin{array}{l}\stackrel{8}{2} \\
2 \\
0 \\
0\end{array}$ & & $\begin{array}{l}n \\
m^{n} \\
1 \\
a^{n}\end{array}$ & $\begin{array}{l}0 \\
2 \\
\alpha \\
\alpha \\
2 \\
m \\
m\end{array}$ & 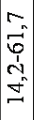 & $\begin{array}{l}\infty \\
\infty \\
0 \\
a \\
\alpha \\
\infty \\
\dot{m}\end{array}$ & & 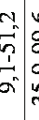 & $\begin{array}{l}0 \\
\Omega^{n} \\
\alpha \\
m^{n} \\
m\end{array}$ & 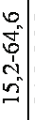 & $\begin{array}{l}\infty \\
\infty \\
0 \\
1 \\
\alpha \\
- \\
m\end{array}$ & $\begin{array}{l}m \\
\sigma \\
\dot{d} \\
\alpha \\
0 \\
0\end{array}$ & $\begin{array}{l}m \\
2 \\
2 \\
\dot{1} \\
\sim \\
2 \\
q\end{array}$ \\
\hline & $\therefore$ & & $\begin{array}{l}\infty \\
\text { N }\end{array}$ & $\begin{array}{l}m \\
\infty \\
\infty\end{array}$ & $\begin{array}{l}m \\
m_{n} \\
m\end{array}$ & $\begin{array}{l}0 \\
i^{n} \\
n^{2}\end{array}$ & & 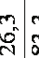 & $\begin{array}{l}m_{n} \\
\infty \\
\infty\end{array}$ & $\begin{array}{l}n \\
m \\
m\end{array}$ & $\begin{array}{l}0 \\
\text { in } \\
n^{2}\end{array}$ & $\frac{\nabla_{n}}{m}$ & $\begin{array}{l}0 \\
\infty^{\circ} \\
2\end{array}$ \\
\hline \multirow{2}{*}{$\bar{n}$} & ì & & $\begin{array}{l}\vec{n} \\
\vec{n} \\
\dot{m} \\
m \\
m \\
m\end{array}$ & 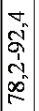 & 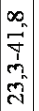 & $\begin{array}{c}\infty \\
\alpha \\
\alpha \\
\alpha \\
\vdots \\
6\end{array}$ & & 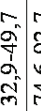 & $\begin{array}{l}r \\
\hat{\alpha} \\
\vdots \\
0 \\
\Sigma \\
\Sigma\end{array}$ & $\begin{array}{c}0 \\
\therefore \\
\bar{i} \\
\vec{m} \\
\vec{m}\end{array}$ & $\begin{array}{l}n \\
n \\
i \\
0 \\
0 \\
n \\
2\end{array}$ & $\begin{array}{l}\infty \\
n \\
n \\
\vdots \\
n \\
\vdots \\
m \\
m\end{array}$ & $\begin{array}{l}1 \\
0 \\
8 \\
0 \\
0 \\
\infty \\
0\end{array}$ \\
\hline & $\overbrace{}^{2}$ & & $\stackrel{m}{\&}$ & $\mid \begin{array}{l}- \\
\infty \\
\infty \\
\infty\end{array}$ & $\begin{array}{l}\vec{n} \\
\mathrm{n}^{\prime}\end{array}$ & $\begin{array}{l}a \\
\tilde{c} \\
\infty\end{array}$ & & $\vec{F}$ & $\begin{array}{l}m_{n} \\
\infty \\
\infty\end{array}$ & $\begin{array}{l}r^{\prime} \\
\delta^{n}\end{array}$ & $\begin{array}{l}n \\
n \\
\infty \\
\infty\end{array}$ & 官 & $\begin{array}{l}+ \\
\infty^{n} \\
\infty\end{array}$ \\
\hline \multirow{2}{*}{ 苞 } & i̊ & & 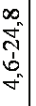 & $\mid \begin{array}{c}m \\
\tilde{c} \\
\tilde{y} \\
b \\
b \\
\infty \\
\infty\end{array}$ & $\begin{array}{c}m \\
c \\
c \\
f \\
b \\
0 \\
\infty \\
\infty\end{array}$ & $\mid \begin{array}{l}0 \\
0 \\
0 \\
0 \\
n \\
\sim \\
n \\
n \\
n\end{array}$ & & 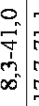 & $\begin{array}{l}\vec{a} \\
\vec{z} \\
\underline{\underline{n}} \\
\vec{E}\end{array}$ & $\begin{array}{l}y \\
y \\
y \\
y \\
1 \\
r \\
\approx\end{array}$ & $\begin{array}{l}r \\
\hat{2} \\
\hat{c} \\
0 \\
\infty \\
\infty\end{array}$ & $\begin{array}{c}0 \\
0 \\
0 \\
\mathbb{T} \\
1 \\
\dot{t} \\
\infty\end{array}$ & 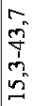 \\
\hline & $\mathrm{d}^{\circ}$ & & $\begin{array}{c}\mathcal{v} \\
\mathfrak{v}\end{array}$ & $\begin{array}{c}\widetilde{c} \\
\widetilde{N} \\
\tilde{N}\end{array}$ & $\begin{array}{c}c \\
\sim \\
N\end{array}$ & $\begin{array}{l}n \\
m \\
m\end{array}$ & & $\begin{array}{l}\vec{v}_{\mathfrak{c}} \\
\vec{c}\end{array}$ & $\begin{array}{l}a \\
\hat{v}\end{array}$ & $\begin{array}{l}n \\
\text { ci } \\
\end{array}$ & : & $\begin{array}{l}\infty \\
n \\
n\end{array}$ & $\begin{array}{l}2 \\
\stackrel{N}{N}\end{array}$ \\
\hline \multirow{2}{*}{ 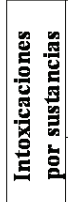 } & i̊ & & $\begin{array}{l}0 \\
0 \\
\infty \\
1 \\
1 \\
\vdots \\
\vdots \\
5\end{array}$ & $\left|\begin{array}{l}0 \\
0 \\
0 \\
0 \\
\infty \\
\infty \\
\hdashline\end{array}\right|$ & 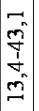 & $\begin{array}{l}0 \\
\hat{a} \\
a \\
\alpha \\
\Rightarrow \\
F\end{array}$ & & 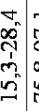 & $\begin{array}{l}\vec{z} \\
\hat{a} \\
\infty \\
n \\
n\end{array}$ & $\begin{array}{l}\vec{n} \\
\overrightarrow{0} \\
1 \\
\overrightarrow{0} \\
0\end{array}$ & 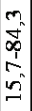 & $\begin{array}{l}0 \\
0 \\
0 \\
1 \\
\sim \\
-1 \\
0 \\
0\end{array}$ &  \\
\hline & & & $\begin{array}{l}a \\
\dot{N}\end{array}$ & $\left|\begin{array}{l}a \\
5 \\
\infty\end{array}\right|$ & $\mid \begin{array}{c}m \\
0 \\
0 \\
i\end{array}$ & $\vec{F}$ & & $\frac{m}{c}$ & $\dot{\infty}^{n}$ & $\begin{array}{l}\infty \\
0 \\
0 \\
m\end{array}$ & $\begin{array}{l}0 \\
\text { in } \\
\text { in }\end{array}$ & $\begin{array}{l}0 \\
\text { N } \\
N\end{array}$ & 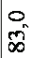 \\
\hline \multirow{2}{*}{ 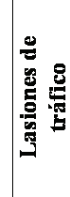 } & 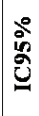 & & $\begin{array}{l}c \\
\infty \\
\infty \\
p \\
i \\
\dot{1} \\
0 \\
c\end{array}$ & $\mid \begin{array}{l}a \\
2 \\
2 \\
\infty \\
\infty \\
\infty\end{array}$ & $\left|\begin{array}{l}0 \\
0 \\
0 \\
0 \\
0 \\
0 \\
\bar{d}\end{array}\right|$ & $\begin{array}{l}n \\
0 \\
\infty \\
0 \\
0 \\
0 \\
0 \\
0\end{array}$ & & 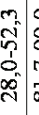 & $\begin{array}{l}a \\
2 \\
\vdots \\
\vdots \\
\infty\end{array}$ & $\begin{array}{l}\nabla \\
n \\
n_{n}^{n} \\
\overbrace{n} \\
n \\
n\end{array}$ & $\begin{array}{l}\infty \\
\infty \\
0 \\
0 \\
0 \\
8 \\
0\end{array}$ & $\begin{array}{l}\vec{n} \\
2 \\
\overrightarrow{2} \\
\overrightarrow{2} \\
\vec{N}\end{array}$ & $\begin{array}{c}r \\
\infty \\
0 \\
i \\
-1 \\
\infty \\
\infty\end{array}$ \\
\hline & 8 & & $\vec{\infty}$ & $\begin{array}{l}a \\
8 \\
a\end{array}$ & $\begin{array}{l}n \\
2 \\
m\end{array}$ & $\begin{array}{c}1 \\
\infty \\
\infty\end{array}$ & & $\stackrel{m}{m}$ & $\begin{array}{l}\nabla \\
\check{8}\end{array}$ & $\begin{array}{l}\overrightarrow{\mathrm{N}} \\
\stackrel{\mathrm{N}}{2}\end{array}$ & $\begin{array}{l}n \\
8 \\
8\end{array}$ & $\frac{n}{m}$ & ने \\
\hline \multirow{2}{*}{ 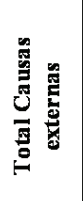 } & ì & & 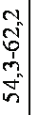 & $\begin{array}{l}- \\
2 \\
2 \\
\infty \\
\infty \\
2 \\
\alpha\end{array}$ & $\begin{array}{l}c \\
1 \\
\infty \\
0 \\
1 \\
0 \\
0 \\
n \\
n\end{array}$ & 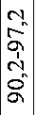 & & 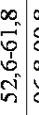 & $\begin{array}{l}\infty \\
\alpha^{n} \\
2 \\
\infty \\
0 \\
0 \\
0\end{array}$ & $\begin{array}{l}5 \\
6 \\
0 \\
0 \\
0 \\
0 \\
n\end{array}$ & $\begin{array}{l}a \\
0 \\
0 \\
\vdots \\
n \\
\vdots \\
a\end{array}$ & $\begin{array}{l}0 \\
1 \\
0 \\
0 \\
n \\
0 \\
0 \\
n\end{array}$ & \begin{tabular}{l}
0 \\
$\infty$ \\
0 \\
0 \\
$\infty$ \\
\multirow{2}{0}{} \\
0
\end{tabular} \\
\hline & $\mathrm{d}^{\mathrm{a}}$ & & $\begin{array}{l}m^{n} \\
\infty^{n}\end{array}$ & 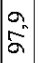 & ci & $\begin{array}{l}\sigma_{n} \\
\dot{\delta}\end{array}$ & & \begin{tabular}{l|l}
$r$ & 0 \\
& 0
\end{tabular} & $\begin{array}{l}a \\
\alpha^{n}\end{array}$ & $\begin{array}{l}\mathbf{N} \\
\tilde{\sigma}\end{array}$ & \begin{tabular}{l}
$r$ \\
\multirow{S}{*}{}
\end{tabular} & in & 8 \\
\hline \multirow{4}{*}{$\frac{\sqrt[3]{3}}{3}$} & 今̊ & & 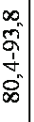 & 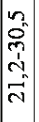 & $\begin{array}{l}n \\
0 \\
0 \\
1 \\
1 \\
8 \\
\infty \\
\infty\end{array}$ & 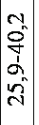 & & $\begin{array}{ll}0 & \\
2 & \\
2 & \\
\vdots & \\
\kappa & \end{array}$ & $\begin{array}{l}\infty \\
0 \\
n \\
n \\
b \\
0 \\
\vdots \\
=\end{array}$ & $\begin{array}{l}n \\
\vdots \\
9 \\
1 \\
m \\
\infty \\
\infty\end{array}$ & 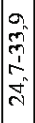 & $\begin{array}{l}\sigma_{n} \\
m^{n} \\
j \\
\dot{\sigma} \\
\infty \\
\infty\end{array}$ & 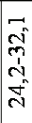 \\
\hline & $0^{2}$ & & $\begin{array}{l}\sim \\
\infty \\
\infty\end{array}$ & $\left|\begin{array}{l}0 \\
n \\
n\end{array}\right|$ & $\begin{array}{l}0 \\
8 \\
8\end{array}$ & $\begin{array}{l}\infty \\
\sim \\
\cdots \\
m\end{array}$ & & $\begin{array}{l}\infty \\
\vdots \\
\infty\end{array}$ & $\begin{array}{l}0 \\
\tilde{N}\end{array}$ & $\begin{array}{l}\infty \\
\infty \\
\infty\end{array}$ & $\begin{array}{l}3 \\
2 \\
2\end{array}$ & $\begin{array}{l}\sim \\
2 \\
\infty\end{array}$ & $\begin{array}{l}0 \\
\infty \\
\infty \\
N\end{array}$ \\
\hline & & & $\sim$ & U & $\infty$ & $\mathscr{U}$ & & $\approx$ & 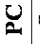 & $\approx$ &  & $\infty$ & $\Xi$ \\
\hline & & : & 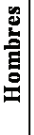 & & 量 & & $=$ & 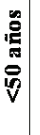 & & 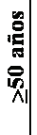 & & $\underset{\xi}{\Xi}$ & \\
\hline
\end{tabular}


En la tabla 3 se observa la matriz de concordancia entre la información procedente del Registro de Mortalidad y la información procedente del IMLC. En términos generales, destacó el elevado número de discordancias entre las causas externas y las enfermedades del sistema respiratorio, y entre las causas externas y las enfermedades del sistema circulatorio. En concreto, destacaron las 53 defunciones codificadas como enfermedades del sistema respiratorio en el Registro de Mortalidad que, considerando la información del IMLC, se codificaron como intoxicaciones. También destacaron los 84 y los 64 fallecimientos codificados en el Registro de Mortalidad como resto de causas externas que se codificaron como suicidio y lesión por tráfico, respectivamente. Asimismo, destacaron las 25 defunciones codificadas en el Registro de Mortalidad en el apartado de trastornos mentales, que se codificaron como intoxicaciones por drogas. Por último, señalar que existió un elevado número de defunciones codificadas como mal definidas en el Registro de Mortalidad que, considerando la información del IMLC, se codificaron principalmente en el grupo de enfermedades del sistema circulatorio o en el grupo de causas externas, concretamente en suicidios o intoxicaciones.

En la tabla 4 se muestra la sensibilidad y el PC según sexo y edad en los grupos de causas externas y mal definidas. Según los grupos de Percy las causas clasificadas como causas subnotificadas en el Registro de Mortalidad son el total de causas externas con valores de sensibilidad de 59,7\% (IC95\%: $56,5-62,9$ ) y de PC de 96,7\% (IC95\%: 94,8$98,0)$ y, concretamente, las lesiones por tráfico, las intoxicaciones y los suicidios con valores de sensibilidad que oscilan entre el $20 \%$ y el $40 \%$ y de PC con valores entre el $80 \%$ y el $95 \%$. Las causas mal definidas presentaron una sensibilidad de 89,2\% (IC95\%: $83,4-93,4)$ y un PC de $28,0 \%$ (IC95\%: $24,2-$ $32,1)$ quedando clasificadas como causas sobrenotificadas en el Registro de Mortalidad. Los homicidios y las caídas, éstas últi- mas con valores de sensibilidad y PC inferiros al $30 \%$, quedaron clasificados como causas con escasa validez. Por último, destacar que no se observaron diferencias estadísticamente significativas ni por sexo ni por grupos de edad.

\section{DISCUSIÓN}

La validez de las estadísticas de mortalidad basadas en los documentos estadísticos (BED y MNP.52) en aquellas defunciones que requieren intervención medicolegal es baja, principalmente por el elevado porcentaje de defunciones clasificadas en el grupo de mal definidas y por la subnotificación de las causas externas, concretamente de las lesiones de tráfico, las intoxicaciones y los suicidios. Las caídas y los homicidios presentan escasa validez. Los resultados no difieren por sexo y grupos de edad.

El índice de concordancia global para todos los grupos de causas fue del 54,8\%, siendo inferior a los obtenidos por De Arán et al. ${ }^{18}$, que utilizaban una muestra de defunciones con intervención medicolegal y las autopsias judiciales como estándar de comparación, y por Pañella et al. ${ }^{14}$ y García Benavides et al. ${ }^{10}$, que utilizaban las historias clínicas. Esta baja concordancia entre los dos registros está relacionada con la inmediatez en la cumplimentación del MNP.52, ya que este documento se suele rellenar antes de disponer del informe definitivo de autopsia. En este sentido, el INE debería mantener abiertos los casos en los que el informe de autopsia no es definitivo, aunque no se conoce el período de tiempo ni los criterios que se siguen para establecerlo. Además, en España, los médicos forenses no tienen la obligación legal de rellenar los documentos estadísticos y los juzgados tampoco incorporan al MNP.52 las conclusiones definitivas de los informes de autopsia o de las pruebas complementarias. Para solventar este problema existen recomendaciones que apuntan a la 
necesidad de una mayor implicación en el proceso de cumplimentación de los documentos estadísticos por parte del médico forense y la implementación de sistemas que permitan la posterior modificación de la información estadística inicialmente referida $^{19}$.

Aunque aproximadamente el 50\% de las defunciones con intervención medicolegal quedan clasificadas en el grupo de causas naturales, éstas sólo representan el 3\% de la mortalidad por causas naturales ocurridas en Barcelona, lo que justifica que el análisis de la validez se haya realizado sólo en el grupo de causas externas y mal definidas. En relación a la subnotificación de las causas externas, coincide con lo hallado por Pañella et al. ${ }^{14}$ y García Benavides et al. ${ }^{10}$, pero no con lo encontrado por De Arán et al. ${ }^{18}$, que clasificaron las causas externas como causas con escasa validez. Sin embargo, es importante señalar que este estudio se realizó para toda Catalunya, lo que implica que los resultados pueden estar influenciados por el entorno rural de ciertas zonas del territorio en un período anterior al de nuestro estudio, lo que hace que los resultados no sean estrictamente comparables.

El hallazgo de la subnotificación de las lesiones por tráfico no coincide con otros estudios $^{14,15,18}$ que utilizaban la historia clínica o las autopsias como estándar de comparación, donde esta causa se consideraba fiable. En el presente estudio la subnotificación es debida al elevado número de defunciones codificadas en el Registro de Mortalidad mediante un código de defunción inespecífico como son las lesiones traumáticas no especificadas (X59).

La subnotificación de las intoxicaciones coincide con un estudio realizado por Brugal et al. ${ }^{11}$ que concluía que el Registro de Mortalidad infraestimaba las defunciones por sobredosis a drogas. En este estudio la subnotificación se explica por la codificación de esta causa en el Registro de Mortalidad como defunciones relacionadas con enfermedades del sistema respiratorio, principalmente como edema agudo de pulmón (J81) o como insuficiencia respiratoria no clasificada en otra parte (J96). Además, es importante señalar que según las normas de la CIE-10 las intoxicaciones por drogas de abuso también pueden clasificarse como trastornos mentales por consumo de drogas (F11-F12, F14-F16, F19). Esto explica las 25 defunciones codificadas en el Registro de Mortalidad como trastornos mentales. En España, este tipo de defunciones se codifican mayoritariamente en los códigos correspondientes al grupo de causas externas, pero en Catalunya se codifica una proporción importante de estas defunciones en el grupo de trastornos mentales por consumo de drogas ${ }^{23}$.

La subnotificación de los suicidios concuerda con lo descrito en la literatura, donde se concluye que las tasas de mortalidad por suicidio están subestimadas ${ }^{24-26}$. En este estudio, la subnotificación de esta causa se explica por el elevado número de defunciones codificadas en el Registro de Mortalidad en el grupo de resto de causas externas con códigos poco específicos, como son las lesiones traumáticas no especificadas (X59) o la obstrucción no especificada de la respiración (W84).

La escasa validez de las caídas coincide con lo hallado en otros estudios ${ }^{14,15,18}$. Ello se explica por el elevado número de defunciones codificadas como lesiones traumáticas no especificadas (X59), como defunciones relacionadas con enfermedades del sistema circulatorio o como suicidios Además, existe un elevado número de defunciones codificadas en el Registro de Mortalidad como caídas que, considerando la información de los informes forenses, se codifican como suicidios, siendo la posible causa de hallar un valor de sensibilidad alrededor del 15\%. Por otra parte, las caídas en personas ancianas habitualmente se producen por fracturas patológicas de cuello de fémur, pero es frecuente no saber 
si la caída produce la fractura (causa externa) o es a la inversa (causa natural). Por esta razón, en muchas ocasiones se emite el CMD codificando la defunción como fractura patológica de cadera o de cuello de fémur y caída. Estas muertes quedan clasificadas en el grupo de causas externas pero no requieren la intervención del IMLC.

Por último, las defunciones codificadas como mal definidas están sobrenotificadas en el Registro de Mortalidad debido principalmente al elevado número de defunciones que considerando la información del IMLC se recodifican en enfermedades del sistema circulatorio o como algún tipo de causa externa.

Aunque existe literatura referente a la validez de la causa básica de defunción en las muertes medicolegales, no se han realizado estudios donde se compare dicha validez por sexo y grupos de edad, se describan los principales patrones de discordancia entre las causas de mortalidad mediante una matriz de concordancia y se identifiquen las principales causas externas con menor validez.

En relación a las limitaciones de este estudio es importante señalar que puede existir una variabilidad entre médicos forenses en la práctica de las autopsias medicolegales lo que introduce una fuente de sesgo interobservador. Aunque en España las indicaciones de la autopsia son homogéneas (se practican al amparo de la Ley de Enjuiciamiento Criminal) puede existir variabilidad a la hora del diagnóstico de las causas y circunstancias de la muerte. Para evitarla sería adecuada la existencia de recomendaciones para el diagnóstico final del caso. Por otra parte, es importante destacar la existencia de variabilidad en las estimaciones de la sensibilidad y del PC debido al reducido número de casos en ciertas causas de defunción, como los homicidios y las caídas. Aún así es importante destacar que se han usado todas las defunciones que requieren intervención medicolegal y no sólo una muestra de ellas. Por último, señalar que los resultados obtenidos para Barcelona no son extrapolables al resto de España.

En conclusión, al analizar las muertes con intervención medicolegal se pone de manifiesto que el Registro de Mortalidad tiene escasa validez en las defunciones por causas externas registradas, siendo las causas más problemáticas las caídas y los homicidios, no habiendo diferencias según sexo y edad. Así, una década después del estudio realizado por De Arán et al. ${ }^{21}$ la situación de la subnotificación de las causas externas en el Registro de Mortalidad se mantiene. Los resultados apuntan a que la cumplimentación del MNP.52 sigue presentado deficiencias en cuanto a la precisión en las causas, ya que raras veces se cumplimenta disponiendo del informe definitivo de autopsia. En el futuro será necesario estudiar si el nuevo Boletín Estadístico de Defunción con Intervención Judicial puede mejorar los resultados encontrados. No obstante la modificación de los documentos estadísticos no parece suficiente si no va acompañada de una mejora en la coordinación entre las distintas instituciones implicadas en el circuito de la información de mortalidad que facilite la incorporación a los Registro de Mortalidad de la información procedente de las fuentes forenses.

\section{AGRADECIMIENTOS}

A las personas del Registro Civil de Barcelona y del Instituto de Medicina Legal de Catalunya que han hecho posible este estudio.

Este artículo forma parte de la tesis doctoral que Mercè Gotsens realiza en la Universidad Pompeu Fabra de Barcelona.

\section{BIBLIOGRAFÍA}

1. Borrell C, Martos D. Cómo cumplimentar correctamente un boletín estadístico de defunción. FMC. 2002; 9:38392. 
2. Arimany-Manso J, Barbería-Marcalain E, Rodríguez Sendin JJ. El nuevo certificado médico de defunción. Rev Esp Med Legal. 2009;36.

3. Teijeira R, Banon R, Hidalgo A, Pradini I. La autopsia médico-legal. Med Clin (Barc). 2006;126:787-92.

4. Crespo AS, Tortosa Lopez JM, Castella GJ, Giménez PD, Sos TP. El parte judicial de defunción. Aten Primaria. $2001 ; 28: 278-82$

5. Instituto Nacional de Estadística. Subdirección General de Censos y Padrón. Proyecto de Reforma de las Estadísticas del Movimiento Natural de la Población. Estadística de Defunciones según la Causa de Muerte. Madrid: INE; 2008 .

6. Crowcroft N, Majeed A. Improving the certification of death and the usefulness of routine mortality statistics. Clin Med. 2001;1:122-25.

7. Lahti RA, Penttila A. The validity of death certificates: routine validation of death certification and its effects on mortality statistics. Forensic Sci Int. 2001;115:15-32.

8. Lunetta P, Lounamaa A, Sihvonen S. Surveillance of injury-related deaths: medicolegal autopsy rates and trends in Finland. Inj Prev. 2007; 13:282-284.

9. Nordrum I, Eide TJ, Jorgensen L. Medicolegal autopsies of violent deaths in northern Norway 1972-1992. Forensic Sci Int 1998; 92:39-48.

10. Benavides FG, Bolumar F, Peris R. Quality of death certificates in Valencia, Spain. Am J Public Health 1989;79:1352-4.

11. Brugal MT, Barrio G, Regidor E, Mestres M, Cayla JA. Discrepancias en el número de muertes por reacción aguda a sustancias psicoactivas registradas en España. Gac Sanit 1999; $13: 82-7$

12. Johansson LA, Westerling R. Comparing Swedish hospital discharge records with death certificates: implications for mortality statistics. Int J Epidemiol. 2000;29:495-502.

13. Johansson LA, Westerling R. Comparing hospital discharge records with death certificates: can the differences be explained? J Epidemiol Community Health. 2002;56:301-8.

14. Pañella H, Borrell C, Rodriguez C, Roca J. Validación de la causa básica de defunción en Barcelona, 1985. Med Clin (Barc). 1989;92:129-34.

15. Rao C, Yang G, Hu J, Ma J, Xia W, Lopez AD. Validation of cause-of-death statistics in urban China. Int J Epidemiol. 2007;36:642-51.
16. Vali M, Lang K, Soonets R, Talumae M, Grjibovski AM. Childhood deaths from external causes in Estonia, 2001-2005. BMC Public Health. 2007;7:158.

17. Vázquez E, Cerdeira S, López de Abajo B, Hervada X. Evaluación de un registro de necropsias extrahospitalarias como instrumento de mejora de la calidad de un registro de mortalidad. Gac Sanit. 1999;13(Supp12):71-126.

18. De Arán BM, Perez G, Rosell J, Molina P. Exactitud de las estadísticas de mortalidad por causas externas y naturales con intervención médico-legal en Cataluña, 1996. Gac Sanit. 2000;14:356-62

19. Xifro-Collsamata A, Pujol-Robinat A, Medalla-Muniz J, Arimany-Manso J. Impacto de los datos utilizados en medicina forense sobre la salud pública. Med Clin (Barc). 2006;126:389-96.

20. Organización Panamericana de la Salud. Clasificación Estadística Internacional de Enfermedades y Problemas Relacionados con la Salud. Décima Revisión. Washington: OPS; 1995.

21. Cohen J. A coefficient of agreement for nominal scales. Educ Psychol Meas. 1960; 20:37-46.

22. Percy C, Stanek E, III, Gloeckler L. Accuracy of cancer death certificates and its effect on cancer mortality statistics. Am J Public Health. 1981;71:242-50.

23. Santos S, Molist G, Barrio G, Pulido J, Bravo MJ, Fernandez-Cuenca $\mathrm{R}$ et al. Codificación de la mortalidad directamente relacionada con drogas ilegales en España: Hacia una adaptación a los criterios estándar europeos. Gac Sanit 2010; 24:309-313.

24. Chishti P, Stone DH, Corcoran P, Williamson E, Petridou E. Suicide mortality in the European Union. Eur J Public Health. 2003;13:108-114.

25. Linsley KR, Schapira K, Kelly TP. Open verdict v. suicide - importance to research. Br J Psychiatry. 2001;178:465-8.

26. Ohberg A, Lonnqvist J. Suicides hidden among undetermined deaths. Acta Psychiatr Scand. 1998;98:214-8. 